\title{
Paclitaxel-Coated Balloon Angioplasty in the Real World: Jack-of-all-Trades?
}

\author{
Ulf Teichgräber ${ }^{1}$ (D)
}

Received: 16 November 2020/ Accepted: 25 November 2020/Published online: 7 December 2020

(C) The Author(s) 2020

Efficacy of drug-coated balloon (DCB) angioplasty is well established for femoropopliteal lesions. However, clinical benefit differs considerably across DCB types [1]. Superiority of Passeo-18 lx DCB over standard balloon angioplasty (POBA) regarding the primary endpoint of 6-month late lumen loss and the secondary endpoint of 6-month binary restenosis had been demonstrated earlier by the BIOLUX P-I randomized controlled trial (RCT). However, the small-scale study had not been powered to compare clinical endpoints, and thus, difference in the incidence of clinically driven target lesion revascularization (TLR) between groups did not reach statistical significance (DCB $15.4 \%$ vs. POBA $41.7 \%, p=0.06$ ) [2]. The BIOLUX P-II RCT on below-the-knee (BTK) lesions showed similar clinical outcomes after DCB angioplasty and POBA.

In this issue of CVIR, Tepe et al. [3] present 24-month results of the real-world BIOLUX P-III registry on performance and safety of infrainguinal Passeo-18 lx DCB angioplasty in 877 participants, providing specific information on femoropopliteal lesions, long lesions $(\geq 15 \mathrm{~cm})$, and in-stent restenosis (ISR). Results on the BTK lesion subgroup had been presented earlier in CVIR. Overall, the study population included a considerably high proportion of participants at increased risk (femoropopliteal subgroup: $43 \%$ diabetes, $33 \%$ renal disease, $41 \%$ current smoker, $35 \%$ critical limb ischemia [CLI], 25\% chronic total occlusions, $17 \%$ severe calcification, and $14 \%$ in-stent restenosis). Mean femoropopliteal lesion length was

Ulf Teichgräber

ulf.teichgraeber@med.uni-jena.de

1 Department of Radiology, Jena University Hospital, Friedrich-Schiller-University Jena, Am Klinikum 1, 07747 Jena, Germany
$96 \pm 78 \mathrm{~mm}$. Investigators predilated $89 \%$ of femoropopliteal lesions with POBA and conducted bailout stenting in $20 \%$. At 24 months, in the femoropopliteal subgroup, primary patency was $67 \%$ (imaging cohort) and freedom from TLR $89 \%$, with considerably worse results in the long lesion- and ISR subgroups, both including BTK lesions.

Previous RCTs reported on 24-month primary patency of $57-90 \%$, and on freedom from TLR of $64-97 \%$ after femoropopliteal DCB angioplasty [1, 4]. However, in most cases, study populations differed considerably from the BIOLUX P-III registry, particularly regarding the share of CLI participants, and thus, direct comparison is not appropriate. However, the AcoArt I [5] trial has some similarities in the study population which may enable a vague orientation on Passeo-18 lx DCB performance. AcoArt I was characterized by comparable proportions of diabetes, CLI, and bailout stenting, but higher shares of long lesions, total occlusions, and ISR. Finally, 24-month primary patency and freedom from TLR in AcoArt I were about the same order of magnitude $(65 \%, 87 \%$, respectively) as in the BIOLUX P-III registry.

In the BIOLUX P-III registry, all-cause mortality in the femoropopliteal subgroup was $10 \%$. Four percent of participants experienced major amputation. Results from the long lesion- and ISR subgroups were slightly worse. In comparison, earlier RCTs on femoropopliteal DCB angioplasty reported 24-month all-cause mortality and incidence of major amputation of $1-13 \%$ and $0-6 \%$, respectively [1, 4]. Thus, BIOLUX P-III safety outcomes were at upper end of range, probably attributed to advanced disease and comorbidities. None of the deaths were adjudicated device or procedure related. Cox regression did not identify paclitaxel dose as predictor of mortality. However, 
whether differences in paclitaxel dose within the study population and total number of deaths were sufficient to assess an effect on mortality might be questioned.

In conclusion, it should be considered that authors provide detailed description of cohort and subgroup characteristics and clinical and imaging outcomes that remarkably contribute to the overall assessment of DCB angioplasty. However, by the nature of registries, the major drawback of BIOLUX P-III is the absence of a control group. Therefore, the actual treatment effect of Passeo18 1x DCB angioplasty and specific interaction effects of subgroups remain uncertain.

Funding Open Access funding enabled and organized by Projekt DEAL. This study was not supported by any funding.

\section{Compliance with Ethical Standards}

Conflict of interest The author declares that he has no conflict of interest with respect to this article.

Consent for Publication For this commentary, consent for publication is not required.

Ethical Approval This article does not contain studies with human participants performed by any of the authors.

Informed Consent For this article informed consent is not required.

Open Access This article is licensed under a Creative Commons Attribution 4.0 International License, which permits use, sharing, adaptation, distribution and reproduction in any medium or format, as long as you give appropriate credit to the original author(s) and the source, provide a link to the Creative Commons licence, and indicate if changes were made. The images or other third party material in this article are included in the article's Creative Commons licence, unless indicated otherwise in a credit line to the material. If material is not included in the article's Creative Commons licence and your intended use is not permitted by statutory regulation or exceeds the permitted use, you will need to obtain permission directly from the copyright holder. To view a copy of this licence, visit http://creativecommons. org/licenses/by/4.0/.

\section{References}

1. Klumb C, Lehmann T, Aschenbach R, Eckardt N, Teichgräber U. Benefit and risk from paclitaxel-coated balloon angioplasty for the treatment of femoropopliteal artery disease: a systematic review and meta-analysis of randomised controlled trials. EClinicalMedicine. 2019;16:42-50.

2. Scheinert D, Schulte KL, Zeller T, Lammer J, Tepe G. Paclitaxelreleasing balloon in femoropopliteal lesions using a BTHC excipient: 12-month results from the BIOLUX P-I randomized trial. J Endovasc Ther. 2015;22(1):14-21.

3. Tepe G, Zeller T, Moscovic M, et al. Paclitaxel-coated balloon angioplasty for the treatment of infrainguinal arteries: 24-Month outcomes in the full cohort of BIOLUX P-III global registry. Cardiovasc Intervent Radiol. 2020. https://doi.org/10.1007/ s00270-020-02663-7.

4. Teichgräber U, Lehmann T, Aschenbach R, et al. Drug-coated balloon angioplasty of femoropopliteal lesions maintained superior efficacy over conventional balloon: 2-year results of the randomized Effpac trial. Radiology. 2020;295(2):478-87.

5. Xu Y, Jia X, Zhang J, et al. Drug-coated balloon angioplasty compared with uncoated balloons in the treatment of 200 chinese patients with severe femoropopliteal lesions: 24-month results of AcoArt i. JACC Cardiovasc Interv. 2018;11(23):2347-53.

Publisher's Note Springer Nature remains neutral with regard to jurisdictional claims in published maps and institutional affiliations. 